\title{
NOMINALISMO E PROGRESSO SCIENTIFICO NELL'ULTIMO MEDIOEVO: GIOVANNI BURIDANO
}

Alessandro Ghisalberti

SÍNTESE - Os pensadores do século XIV, até poucos anos, eram considerados como críticos destruidores, que minaram as bases do saber elaborado durante o século XIII. Nos últimos anos, mostrou-se que esta idéia é falsa. 0 "nominalismo" de homens como Ockham, Gregório de Rimini, João Buridano e outros, representa um momento importante na história da ciência, graças principalmente à aplicação de sua refinada lógica na releitura da física e da cosmologia. Com a revisão global da linguagem filosófi$\mathrm{ca}$, eles criaram novas linguagens, e nisto encontra-se sua contribuição para a história da ciência. Foi assim que Buridano inovou em questões de física, como na noção do espaço e do movimento.
ABSTRACT - Until some years ago the thinkers of the fourteenth century were considered as destroying critics undermining the bases of the knowledges elaborated during the thirteenth century. However recent studies have shown that this idea is wrong. The "nominalism" of men like Occam, Gregory of Rimini, John Buridan and others represents an important moment in the history of the science, mainly because it has applied its refined logic to a new interpretation of physics and cosmology. Revising the whole philosophical language, the "nominalists" have created new languages and contributed to the history of the science. Thus Buridan, for instance, has brought innovations in the realm of physics related to the notions of space and movement.

\section{1 - La scolastica, l'aristotelismo e la scienza}

Nei manuali della prima metà del sec. XX, il nominalismo è stato costantemente presentato come un periodo di eccessivo criticismo, che ha minato le solide basi del sapere scolastico edificato dai maestri del sec. XIII; critici oppositori della metafisica, i maestri del sec. XIV erano qualificati 'nominalisti' perché avrebbero elaborato una logica che assegnava all'universale solo una valenza concettuale $e$ nominale, legata cioè alla capacità dei termini universali di rinviare a una pluralità di cose, in se stesse tutte singolari.

A partire dal secondo dopoguerra, la storiografia relativa al tardo medioevo ha respinto l'accezione puramente negativa del nominalismo: esso va correttamente

Università Cattolica di Milano.

\begin{tabular}{|l|l|l|l|l|l|} 
VERITAS & Porto Alegre & v. 42 & $\Pi^{\underline{0}} 3$ & Setembro 1997 & p. 679-687 \\
\hline
\end{tabular}


inteso come un terminismo rigoroso, attento alle valenze logico-linguistiche del linguaggio umano, che è costituito da segni (mentali, orali o scritti) attraverso i quali passa ogni discorso 'scientifico', sia esso metafisico o fisico, teologico o retorico-poetico.

Parlando della scienza in età nominalistica, ci si muove all'interno della cultura medievale, definita 'scolastica' perché cresciuta e sviluppata nelle scuole di vario ordine e grado, cultura che si distingue da quella delle epoche successive soprattutto, in campo scientifico, per avere avuto a disposizione un numero limitato e preciso di fonti: la Bibbia e i trattati scientifici dell'antichità classica. La ricezione di queste opere avvenne gradualmente: per molti secoli, e cioè fino al sec. XII, l'occidente latino non conobbe le opere scientifiche di Aristotele e degli aristotelici arabi, e fondò le proprie conoscenze scientifiche fondamentalmente sui testi di questi quattro autori della tarda antichità: Severino Boezio, Cassiodoro, Isidoro di Siviglia e Beda il Venerabile. Redigendo manuali ed enciclopedie del pensiero scientifico dell'ultima classicità, essi hanno trasmesso al medioevo i contenuti dello scibile articolato nelle sette arti liberali, aggiungendovi elementi di cosmografia biblica e la trattazione di argomenti minori circa gli utensili casalinghi e gli svaghi.

Nel sec. XII un rinnovato interesse per la natura dilata lo studio della cosmologia: soprattutto alla scuola di Chartres, i maestri avallano una lettura del Timeo, il dialogo platonico che tratta dell'origine del mondo, in modo da trovarne un profondo accordo con il libro della Genesi, che contiene il racconto biblico della creazione del mondo. Il Dio della Bibbia è riconoscibile negli attributi che Platone assegna al demiurgo.

Alla fine del sec. XII e agli inizi del sec. XIII si diffondono le prime traduzioni latine della Fisica e del De caelo di Aristotele, oltre che dei trattati astronomici di Tolomeo (sec. II d. C.), i quali proponevano una teoria dei moti e dei luoghi che escludeva l'esistenza fuori dal cielo del luogo, del moto e del tempo: il luogo, per essere tale, deve sempre contenere un corpo; il vuoto è per definizione ciò in cui non è presente alcun corpo, e pertanto non è nulla, non può esistere; il tempo, essendo la misura del moto, può trovarsi solo dove c'è un corpo naturale.

Sempre nella visione aristotelico-tolemaica l'universo ha forma sferica, poiché la sfera è il corpo formalmente più perfetto, e consta di un sistema di sfere omocentriche: la prima sfera dall'alto è quella delle stelle fisse, così denominata perché le stelle, benché si muovano insieme con la sfera che le contiene, mantengono fra di loro sempre la medesima distanza. Questa sfera riceve il proprio movimento da una causa esterna, il primo motore immobile, e perciò possiede il movimento più veloce e regolare.

Al di sotto di essa sono disposte altre sette sfere concentriche, alle quali sono infissi altrettanti pianeti, secondo questo ordine dall'alto: Saturno, Giove, Marte, Mercurio, Venere, Sole, Luna. La terra occupa il luogo più in basso del cosmo; è al centro dell'universo, perfettamente immobile, avente forma sferica. Le leggi della fisica sono segnate dalla natura degli elementi fondamentali, che sono acqua, terra, aria, fuoco; le forme dei quattro elementi sono contrassegnate dalle determinazioni delle quattro qualità prime ed originali: caldo, freddo, secco, umido; esse 
hanno carattere attivo e determinano i mutamenti qualitativi: l'acqua è fredda e umida; la terra è fredda e secca; l'aria è calda e umida; il fuoco è caldo e secco. Le altre qualità sensibili (pesante-leggero, denso-raro, ruvido-liscio, duro-molle) sono qualità seconde, ossia derivate dalle prime.

La fisica "qualitativa" aveva avuto una rigorosa sistemazione nelle opere di Aristotele e venne accolta sempre, anche dai maestri del sec. XIII, come la teoria portante in tutto il campo della scienza, ossia anche nelle discipline diverse dalla cosmologia e che gli scolastici si sforzavano di riproporre, quali la medicina, l'astrologia-astronomia, l'alchimia. Questo quadro delle conoscenze scientifiche dell'ultimo medioevo è indispensabile al fine di comprendere la diversità dei maestri denominati 'nominalisti' riguardo alla lettura dei testi di Aristotele e alla valutazione delle dottrine scientifiche greco-arabe.

\section{2 - Il rinnovamento nominalistico della filosofia della natura}

La genesi della revisione critica delle dottrine fisiche e cosmologiche ereditate dalla classicità è riconducibile a due fattori che diventarono operativi a partire dai primi decenni del sec. XIV, il primo di carattere logico-linguistico, il secondo legato alla metodologia teologica. Ne furono coinvolti tutti i pensatori del tempo: Giovanni Duns Scoto e la vivace, numerosa schiera degli 'scotisti'; Guglielmo di Ockham, cui la tradizione successiva accorderà la primogenitura nella scuola dei nominalistae; Gregorio da Rimini, teologo agostiniano; Tommaso Bradwardine e i calculatores del Merton College; Giovanni Buridano, Nicola Oresme e Marsilio di Inghen, che applicarono la loro raffinata logica nella rilettura della fisica e della cosmologia.

Circa il fattore che abbiamo chiamato logico-linguistico, l'influenza sullo sviluppo della filosofia della natura tardo-medievale venne dai nuovi linguaggi dell'astrologia, dell'alchimia, dei calcoli e delle misure nell'indagine dei fenomeni fisici: si pensi al linguaggio sull'intensione e la remissione delle forme, che coinvolse molte problematiche teologiche, al linguaggio sul primo e sull'ultimo istante dell'essere (o del non-essere) dell'ente, al linguaggio sull'infinitamente divisibile o sulle proporzioni.

Il linguaggio dei nominalisti riflette il rigore derivante dall'analisi logica molto serrata sullo status delle verita universali e necessarie della scienza, come dalle questioni sulle caratteristiche dell'evidenza e della certezza. Se nel sec. XIII il dibattito sull'ente e sui principi che costituiscono gli oggetti che popolano l'universo, a partire dal principio creatore di tale universo, aveva avuto un carattere fondamentalmente metafisico, proiettato sullo statuto ontologico della realtà, nel śec. XIV il dibattito maturò una consapevolezza nuova: in prima istanza si trattava di "oggetti logici", di elementi che riguardavano primariamente la conoscenza e perciò occorreva innanzitutto dirigere l'attenzione su di essi come su proposizioni, o come su termini che firmano proposizioni, e sulle relazioni che si possono stabilire tra proposizioni. In questa direzione va ricordato il dibattito sviluppatosi nell'ockhamismo (soprattutto con Ockham e Gregorio da Rimini) sullo statuto della scienza dimostrativa, che implicava il dibattito sul requisito dell'universalità e della necessità delle proposizioni in rapporto alla radicale con- 
tingenza del mondo naturali, oltre al dibattito su ciò che propriamente forma l'oggetto di una proposizione scientifica.

Dall'analisi logica del linguaggio si arrivò così al metalinguaggio: se le proposizioni sono le uniche entità che possono essere dette universali, necessarie, sempre vere, allora parlare di scienza, ossia interrogarsi sull'epistemologia scientifica, significa parlare delle proposizioni e dei loro costitutivi, significa usare il metalinguaggio, termine questo che, in senso largo, indica ogni discorso intorno a proposizioni, a termini a ad altre entità logiche, a differenza del linguaggio che parla invece delle cose, della realtà. Per esempio, la proposizione: "Questo è il primo istante dell'esistenza di Socraten, può avere un'analisi linguistica che bada alla realtà, all'oggettività dell'asserto, che può esprimersi nel fissare cronologicamente nella realtà il primo istante di Socrate. Può avere altresi un'analisi metalinguistica, che potrebbe tradursi in questi asserti: "Le seguenti proposizioni sono vere: 'Socrate esiste nell'istante presente' e 'Socrate non esisteva prima dell'istante presente' '. Il metalinguaggio è riconoscibile dall'enfasi che l'analisi pone sul fatto che si sta parlando di proposizioni o di termini, e non c'è un nudo enunciato de re; ciò risultava di estrema utilità nella spiegazione della scienza aristotelica segnata dal carattere dell'universalità e della necessità, da parte di autori che sostenevano il primato del singolare, con il connesso carattere particolarista dell'ontologia. Per rendere compatibile la filosofia aristotelica della natura con l'affermazione secondo cui esistono solo res individuae, solo sostanze individuali e qualità individuali, lo strumento metalinguistico era idoneo a spiegare come i termini concreti, non aventi una corrispondenza con un preciso individuo nella realtà, siano trattabili attraverșo più proposizioni. Per esempio, il termine concreto 'bianco', nella proposizione "Socrate è bianco": per verificare tale proposizione, dobbiamo risolverla in queste due altre proposizioni, ciascuna delle quali puo essere vera: "Socrate esisten e "la bianchezza inerisce a Socrate".

La stessa procedura, ossia il ricorso a più proposizioni per spiegare una proposizione, si applica ai termini relativi, a quelli collettivi e ai nomi connotativi astratti come lunghezza, misura, tempo, moto: questi termini si possono ridurre a proposizioni esplicative, che mettano in risalto come la predicazione non concerne direttamente degli enti reali, ma delle intenzioni, dei termini, per cui nella proposizione: «il moto è nel mobilen, l'è (est) non va inteso come se significasse: esiste realmente, bensì come equivalente di essere predicato ("il moto è predicato nel mobilen). Il discorso, a questo punto, si specializzava, con l'uso del metalinguaggio nella logica proposizionale (soprattutto nelle proposizioni della possibilità), nella definizione dei termini negativi come la privazione, la tenebra, il nulla, nell'analisi delle nozioni fisiche di indivisibile, di continuo, di infinito, di punto, di linea, di istante, di vuoto. Di tutte queste cose, osservava Ockham, è possibile parlare solo "mediantibus vocibus, vel conceptibus, vel aliis signis".

Come secondo fattore di rinnovamento della scienza nel sec. XIV va ricordata la metodologia teologica: le condanne dell'aristotelismo ad opera di Stefano Tem- 
pier nel 1277 hanno avuto molta incidenza sull'epistemologia scientifica, per le continue implicazioni di temi connessi con l'onnipotenza di Dio.'

\section{3 - Le novità di Buridano nel campo della fisica}

Dopo le condanne di Tempier, i pensatori cristiani sentirono l'esigenza di distinguere tra la natura intrinsecamente contingente del cosmo, i cui limiti sono stabiliti dall'onnipotenza divina, e la portata delle leggi naturali del cosmo, cosi come oggi è strutturato. Buridano recepisce questa urgenza, distinguendo l'impossibile secundum naturam dall'impossibile simpliciter: ciò che appare impossibile secondo le leggi naturali non è tale in assoluto, in rapporto cioè alla sovrana potenza di Dio creatore. Questa distinzione diventa lo strumento privilegiato per introdurre ipotesi nuove, fuori dal vincolo della fisica aristotelica, quali la possibilità dello spazio tridimensionale vuoto, la possibile esistenza di altri mondi, l'eliminazione delle sostanze separate come motori delle sfere celesti.

\section{1 - Il problema dello spazio}

Essendo primariamente contrassegnata dalla categoria della quantità, la sostanza corporea risulta estesa nello spazio secondo le tre dimensioni: lunghezza, larghezza, profondità; ogni sostanza materiale è perciò estesa, ossia le sue parti sono situate spazialmente in modo continuo l'una dopo l'altra. Inoltre il moto naturale degli elementi verso $i$ loro luoghi naturali mostra la capacità dello spazio di esercitare una certa potenza: gli elementi pesanti infatti (terra e acqua) tendono verso il basso, e quivi si trovano in quiete. L'idea di luogo è quella di "limite del corpo che contiene", ossia quando un corpo è in un luogo, significa che è contenuto in un altro corpo, col quale è in contatto immediato: la sua superficie esterna combacia in tutti i suoi punti con la superficie interna del corpo che lo contiene e lo limita, e questo corpo è il luogo proprio del corpo contenuto. Nella concezione aristotelica perciò lo spazio risulta essere l'insieme dei luoghi propri dei corpi; esso non può essere infinito, perché il cielo rappresenta il luogo contenente tutti i corpi sublunari. Viene inoltre esclusa l'esistenza del vuoto, perché la superficie interna del corpo che contiene e la superficie esterna del corpo contenuto devono combaciare.

Nelle Quaestiones de caelo Buridano distingue l'ordine naturale dall'ordine della pura possibilità, e giunge ad eliminare, sia pure a livello di ipotesi, uno dei principali ostacoli che Aristotele aveva addotto contro l'esistenza di uno spazio tridimensionale vuoto, ossia l'impossibilità per le dimensioni dei corpi di compenetrarsi o di coesistere in un medesimo spazio: "Anzitutto affermo che non si deve porre per via naturale uno spazio fuori del cielo; infatti esso sarebbe una dimensione avente lunghezza, larghezza e profondità, e tale dimensione sarebbe o sostanza o accidente. Naturalmente parlando non può dirsi sostanza, perché il filosofo naturale deve ammettere solo una sostanza corporea che si muove di moto circolare,

Cfr. A. Ghisalberti, Fisica aristotelica e onnipotenza di Dio in Guglielmo di Ockham, in Veritas, 41 (1996), pp. 483-492; Id., Giovanni Buridano dalla metafisica alla fisica, Milano 1975, pp. 130-207. 
e questa appartiene alla natura celeste, perciò non è fuori dal cielo, oppure una sostanza che si muove naturalmente verso l'alto o verso il basso, e i filosofi naturali la collocherebbero sotto il cielo; dunque il filosofo naturale non deve ammettere, in base alla scienza naturale, una sostanza corporea al di là del cielon.'

Diversa è la risposta se ci si colloca a livello della pura possibilità, stabilita in rapporto all'onnipotenza di Dio: «Per la potenza divina infatti non è assurda la penetrazione di un corpo, perciò Dio con ogni corpo mobile potrebbe nello stesso luogo formare un'altra dimensione uguale secondo la penetrazione delle dimensionir."

Analogamente si avanza l'ipotesi della possibilità del vuoto, sempre sulla base dell'onnipotenza divina: Aristotele escludeva che Dio possa muovere il mondo di moto rettilineo, perché fuori del luogo che contiene il mondo non vi è alcun altro corpo capace di contenerlo. Questo vincolo, osserva Buridano, non può valere nell'ipotesi di un intervento divino: "Non si richiede un luogo perché qualcosa sia mosso di moto rettilineo dalla potenza divina... Anzi, Dio potrebbe anche liberare questa pietra dal luogo: se annullasse tutti gli altri corpi, lasciando questa pietra, questa pietra sarebbe in un luogo, anzi non ci sarebbe luogo alcuno. Eppure Dio potrebbe muovere questa pietra di moto rettilineo, esattamente come può muovere il.mondo interon. ${ }^{4}$

Uttima conseguenza di rilievo è l'ammissione della possibile esistenza di più mondi: ogni vincolo fisico, connesso con la concezione aristotelica dell'universo perfetto nelle sue parti e chiuso nella sua struttura, cade di fronte all'ipotesi dell'intervento soprannaturale di Dio. Cade in particolare la preclusione aristotelica circa la necessità che la terra attuale debba essere il centro di un altro ipotetico universo: non è vero, osserva Buridano, che la terra attuale possieda in sé la ratio di centro, per cui tutti i centri debbano convenire omogeneamente con essa. Ogni mondo ha un suo centro naturale e perciò la terra di un ipotetico altro mondo starebbe naturalmente in quel centro, e non occuperebbe il centro del mondo attuale.

\section{2 - Il problema del moto e l'impetus}

Per Aristotele ogni movimento deve avere una causa che coesista con il corpo mosso e renda in tal modo ragione del passaggio dalla potenza all'atto che il movimento comporta. La causa del movimento può essere intrinseca e 'naturale', ossia il moto dipende dalla natura o forma del corpo, come nel caso del moto naturale degli elementi, dove la pesantezza e la leggerezza sono cause naturali rispettivamente del moto del pesante verso il basso e del leggero verso l'alto. La causa può anche essere estrinseca, rappresentata da un elemento che muove il corpo, accompagnandosi con questo per tutta la durata del movimento. Nel caso del moto violento di un proiettile, di un corpo pesante che percorre un tragitto dal

" G. Buridano, Il cielo e il mondo. Commento al Trattato "Del cielo" di Aristotele, introd., trad. it. e note di A Ghisalberti, Milano 1983; I, XVI, pp. 181-182.

Tbid., I, XV, p. 171.

Ibid., I, XVI, p. 179. 
basso in alto oppure attraverso l'aria, ci si chiede quale sia la causa di tale moto, dopo che il proiciente ha lasciato il proiettile (la freccia lanciata dall'arco, la pietra scagliata).

La risposta di Aristotele ricollegava il moto violento del proiettile all'aria, la quale può muovere in due modi: o esercitando una spinta, quando essa sopraggiunge per colmare il vuoto che il proiettile ha lasciato con il suo spostamento (teoria dell'antiperistasi); oppure trascinando con sé il proiettile, in forza della spinta che l'aria circostante il proiettile ha ricevuto dal lanciatore al momento del lancio. La teoria dell'antiperistasi, già criticata da Aristotele, viene respinta da Buridano per le difficoltà sollevate dall'esperienza: i corpi che si muovono circolarmente, e perciò non lasciano alcun vuoto dietro di sé, come nel caso di una mola o di una ruota, continuano nel loro movimento anche dopo che la spinta è cessata.

L'altra spiegazione, che attribuisce la trasmissione di una spinta all'aria circostante il proiettile per opera del lanciatore, urta parimenti contro dei dati di esperienza: se si isola, mediante un panno, l'aria contigua di una ruota, dopo che colui che muoveva ha cessato l'azione, non per questo la ruota si fermerà. Analogamente, se una nave venisse avvolta da teli e spinta velocemente in un corso d'acqua, qualora si ritirassero i teli e si arrestasse l'aria vicina, non per questo la nave si fermerà. D'altronde tutti possono constatare che se si spinge contro una persona l'aria, senza la pietra, con tutta la velocità possibile ad una mano, non succede che quella spinta dell'aria venga avvertita; "E ancora: perché non puoi lanciare una piuma per uno spazio di cinque piedi? Se fosse l'aria spinta a muovere il proiettile, quell'aria dovrebbe muovere di più la piuma, e con più facilità rispetto a una pietra pesanten..

Queste 'apparenze' non vengono spiegate dalle teorie tradizionali; il fatto stesso che Buridano le abbia descritte analiticamente testimonia la rilevanza che esse hanno avuto nell'indirizzarlo alla ricerca di soluzioni nuove e insieme il peso che l'esperienza ha avuto nelle sue analisi fisiche, per arrivare a proporre una nuova soluzione. Buridano prospetta la possibilità che il proiettile, dopo che ha lasciato il lanciatore, sia mosso da uno slancio o impetus, direttamente proporzionale alla forza motrice iniziale e alla quantità di materia del proiettile. Tale impetus aumenta con la velocità del moto e diminuisce per opera della resistenza sviluppata dagli elementi (dall'aria).

Già intravista da Francesco della Marca (intorno al 1326), la dottrina dell'impetus riceve da Buridano una nuova ed organica sistemazione: oltre che nella spiegazione del moto violento il maestro parigino ricorre all'impetus anche per spiegare l'accelerazione dei gravi in caduta libera. Buridano infatti ritiene smentita dall'esperienza la spiegazione data da Aristotele, secondo cui la maggiore velocità del moto del grave (qualsiasi corpo pesante) quando si avvicina alla fine della caduta è causata dalla maggiore vicinanza con il luogo naturale. "Occorre immaginarsi che il corpo pesante riceva dal suo motore principale, ossia dalla gravità, non solo il moto, ma insieme con questo anche un certo slancio (impetus) che ha la capacità di muovere lo stesso corpo pesante, permanendo la

Ibid., II, I, pp. 419-420. 
gravità naturale. Siccome quello slancio si acquisisce comunemente con il moto, perciò quanto più il moto è veloce, maggiore e più forte è quello slancio. Pertanto all'inizio il corpo pesante è mosso solamente dalla sua gravità naturale, perciò si muove lentamente; in seguito è mosso dalla stesa gravità e dallo slancio acquisito, contemporaneamente, perciò si muove più velocementen. ${ }^{6}$

Una terza, rilevante applicazione della teoria dell'impetus riguarda il moto delle sfere celesti. Come è noto, la tradizione aristotelico-tolemaica ricorreva a delle intelligenze motrici, sostanze spirituali aventi il compito di causare il movimento dei corpi celesti, il cui moto non tende a luogo alcuno e perciò non può essere naturale. Buridano, nelle questioni sulla Fisica" e in quelle sulla Metafisica, ${ }^{8}$ osserva come il ricorso alle intelligenze per spiegare il moto dei cieli non abbia un fondamento esplicito nel testo biblico; ritiene perciò possibile 'immaginare' (una imaginatio) che le sfere celesti siano mosse dall'impetus impresso da Dio nel momento della creazione. Siccome il moto circolare non incontra resistenze, le sfere continuerebbero a muoversi in forza dell'impetus ricevuto inizialmente e secondo la velocità posseduta nel momento in cui Dio ha interrotto la spinta. In questo modo il maestro parigino ha compiuto il primo passo verso l'affermazione di un unico sistema di leggi che governano la meccanica dell'universo fisico, sia celeste sia sublunare, ipotizzando l'abbandono della credenza nella natura divina delle potenze motrici del cielo e muovendo la ricerca nella direzione di quello che più tardi sarà chiamato il principio d'inerzia.

\section{4 - Dalla scienza tardomedievale alla moderna rivoluzione scientifica}

È indubbio che le novità sia dello studio scientifico della natura sia della rilettura critica dell'aristotelismo operata nel sec. XIV vanno in direzione della rivoluzione scientifica, che nel sec. XVI porterà alla nascita della moderna scienza empirica induttiva, all'abbandono della fisica qualitativa e della concezione del mondo chiuso connessa al geocentrismo.

Nel passato però si è troppo insistito sulla continuità fra la scienza dell'ultimo medioevo e la fisica galileiana; Pierre Duhem, ${ }^{3}$ Alistair C. Crombie ${ }^{10}$ ed Edward Grant" hanno sottolineato la vicinanza delle intuizioni e delle ipotesi dei medievali alle scoperte di Copernico, Francesco Bacone, Galileo e Newton, a proposito della meccanica e della cinematica. Molto più cauto è invece il giudizio espresso da Alexandre Koyré, ${ }^{12}$ Anneliese Maier ${ }^{13}$ e, tra i viventi, da John Murdoch:" la princi-

\footnotetext{
Ibid., II, XII, p. 326.
}

Cfr. G. Buridano, In VII Physicorum, q. 12.

Cfr. G. Buridano, In XII Metaphysicorum, q. 9.

Cfr. P. Duhem, Le système du monde, I-V, Hermann, Paris 1913-1917, rist. 1954; VI-X, Hermann, Paris 1954-1959; Id., Études sur Léonard de Vinci: ceux qu'il a lu, ceux qui l'ont lu, 3 voll., Hermann, Paris 1906-1913, rist. 1955.

${ }^{10}$ A. C. Crombie, Da S. Agostino a Galileo. Storia della scienza dal V al XVII secolo (prima edizione, New York 1952), tr. it., Feltrinelli, Milano 1970 (con ampia bibliografia).

1 Edward Grant, La scienza nel medioevo (prima ediz., Cambridge 1971), tr. it. I Mulino, Bologna 1983 (con bibliografia ragionata).

12 Cfr. A. Koyré, Études d'histoire de la pensée scientifique, PUF, Paris 1966; Dal mondo chiuso all'universo infinito, tr. it. Feltrinelli, Milano 1970. 
pale novità del sec. XIV non è stata la meccanica, bensì la revisione globale del linguaggio filosofico, con la creazione dei nuovi linguaggi della matematica, della logica, della teologia, che hanno aperto nuiove piste di ricerca in tutti i settori dello scibile. Non è perciò possibile ridurre la storia del pensiero scientifico medievale a premessa della storia dell'evoluzione della meccanica del Seicento. L'originalità della stagione terdomedievale è da ricondurre alla generale revisione della metodologia di ricerca: si moltiplicano i casi da analizzare, diventa urgente l'istanza di trovare gli strumenti e il linguaggio che misurino l'ordine fisico ed i suoi cambiamenti, che rendano ragione dei requisiti del discorso scientifico, che si facciano carico di una metodologia interdisciplinare. Paradigmatica, in questa direzione, è la distinzione tra la potenza assoluta e la potenza ordinata in Dio, che permette di prendere in considerazione ipotesi improponibili nell'ambito dell'aristotelismo così come, in teologia, consente di affinare il discorso sulla trascendenza di Dio, sulla natura dei 'meriti' dell'uomo nei confronti della grazia e della salvezza, sullo scarto tra l'infinito risultante dalla somma di illimitate qualità finite e l'infinito che è tale in forza della sua immensità e assolutezza.

La stagione nominalista non si impone all'attenzione degli studiosi per ciò che ha preparato, ma per quello che in sé stessa è stata: da un lato, essa ha utilizzato l'aristotelismo, e per questo appartiene alla stagione scolastica della scienza; dall'altro lato ha espresso un uso dell'aristotelismo che ne vincesse il carattere frenante nei confronti del rinnovamento della scienza, rendendolo uno dei luoghi privilegiati della ricerca di una nuova concezione della natura. Questo è stato reso possibile dalla logica, dal linguaggio filosofico e scientifico, dall'uso del metalinguaggio, dal rinnovamento della metodologia teologica, tutte conquiste formidabili ed originali dei maestri del sec. XIV.

${ }^{13}$ Cfr. A. maier, Studien zur Naturphilosophie der Spätscholastik, 5 voll., Edizioni di Storia e Letteratura, Roma 1949-1958. Un'antologia di studi tratti da questi volumi, tradotti in italiano, è stata curata da M. Parodi e A. Zoerle: cfr. A. Maier, Scienza e filosofia nel medioevo. Saggi sui secoli XIII e XIV, Jaca Book, Milano 1984. I saggi qui tradotti vertono su alcuni tipici problemi di filosofia naturale, quali il tempo, il continuo, il rapporto necessità-caso, il problema della costituzione dei corpi misti.

${ }^{14}$ Cfr. J. E. Murdoch, "Rationes Mathematice": Un Aspect du Rapport des Mathématiques et de la Philosophie au Moyen Âge, Paris 1962; "Scientia mediantibus vocibus". Metalinguistic Analysis in Late Medieval Natural Philosophy, in Autori Vari, Sprache und Erkenntnis im Mittelalter (Atti del VI congresso internazionale di Filosofia medievale, Bonn, 29 agosto - 3 settembre 1977), "Miscellanea Mediaevalia" 13/1, Berlin-New York 1981, pp. 73-106; The Involvement of Logic in Late Medieval Natural Philosophy, in Studies in Medieval Natural Philosophy, ed. by S. Caroti, Firenze 1989, pp. 328. 